\title{
Effects of Dynamic Fragmentation on the Impact Force Exerted on Rigid Barrier: Centrifuge Modelling
}

\begin{tabular}{|r|l|}
\hline Journal: & Canadian Geotechnical Journal \\
\hline Manuscript ID & cgj-2018-0092.R2 \\
\hline Danuscript Type: & Article \\
\hline Author: & $19-$ Nov-2018 \\
\hline $\begin{aligned} \text { Complete List of Authors: } \\
\text { Kabmitted by the }\end{aligned}$ & $\begin{array}{l}\text { Environmental Engineering } \\
\text { Choi, Clarence; Hong Kong University of Science and Technology, } \\
\text { Department of Civil and Environmental Engineering } \\
\text { Cheung, Desmond Ka Ho; Hong Kong University of Science and } \\
\text { Technology, Department of Civil and Environmental Engineering } \\
\text { Cui, Yifei; Hong Kong University of Science and Technology, Department } \\
\text { of Civil and Environmental Engineering }\end{array}$ \\
\hline $\begin{array}{r}\text { Is the invited manuscript for } \\
\text { consideration in a Special } \\
\text { Issue? : }\end{array}$ & $\begin{array}{l}\text { Advances in Landslide Understanding } \\
\text { modelling }\end{array}$ \\
\hline &
\end{tabular}

\section{SCHOLARONE Manuscripts}




\section{General information of the article}

Type of paper: Article

Title: Effects of dynamic fragmentation on the impact force exerted on rigid barrier:

centrifuge modelling

Authors: Charles Wang Wai Ng, Clarence Edward Choi*, Desmond Ka Ho Cheung, and Yifei Cui

\section{Author information:}

Co-author: Charles Wang Wai Ng

Chair Professor, Department of Civil and Environmental Engineering, Hong Kong University of Science and Technology, Clear Water Bay, Kowloon, Hong Kong.

E-mail: charles.ng@ust.hk

\section{Corresponding author*: Clarence Edward Choi}

Research Assistant Professor, Department of Civil and Environmental Engineering, and Institute for Advanced Study, Hong Kong University of Science and Technology, Clear Water Bay, Kowloon, Hong Kong.

E-mail: ceclarence@ust.hk

\section{Co-author: Desmond Ka Ho Cheung}

PhD candidate, Department of Civil and Environmental Engineering, Hong Kong University of Science and Technology, Clear Water Bay, Kowloon, Hong Kong E-mail: khcheungal@connect.ust.hk

\section{Co-author: Yifei Cui}

Research Assistant Professor, Department of Civil and Environmental Engineering, Hong Kong University of Science and Technology, Clear Water Bay, Kowloon, Hong Kong E-mail: yifeicui@ust.hk

Number of words in abstract: 211

Number of words in main text: 7223

Number of tables: 3

Number of figures: 9 


\title{
Effects of Dynamic Fragmentation on the Impact Force Exerted
}

\section{on Rigid Barrier: Centrifuge Modelling}

\author{
C. W. W. Ng${ }^{1}$, C. E. Choi ${ }^{12^{*}}$, D. K. H. Cheung ${ }^{1}$, and Y. Cui ${ }^{1}$ \\ Department of Civil and Environmental Engineering; the Hong Kong University of Science and Technology, Hong Kong 1 \\ The HKUST Jockey Club Institute for Advanced Study, Hong Kong ${ }^{2}$ \\ Corresponding author*
}

\begin{abstract}
Bi-dispersity is a prerequisite for grain-size segregation, which transports the largest particles to the flow front. These large and inertial particles can fragment upon impacting a barrier. The amount of fragmentation during impact strongly influences the force exerted on a rigid barrier. Centrifuge modelling was adopted to replicate the stresses for studying the effects of bi-dispersity in a granular assembly and dynamic fragmentation on the impact force exerted on a model rigid barrier. To study the effects of bi-dispersity, the ratio between the diameters of small and large particles $\left(\delta_{\mathrm{s}} / \delta_{1}\right)$, characterising the particle size distribution (PSD), was varied as $0.08,0.26$, and 0.56 . The volume fraction of the large particles was kept constant. An $\delta_{\mathrm{s}} / \delta_{1}$ tending towards unity characterises inertial flow that exerts sharp impulses, and a diminishing $\delta_{\mathrm{s}} / \delta_{1}$ characterises the progressive attenuation of these sharp impulses by the small particles. Flows dominated by grain-contact stresses $\left(\delta_{\mathrm{s}} / \delta_{1}<0.26\right)$, as characterised by the Savage Number, are effective at attenuating dispersive stresses of the large particles, which are responsible for reducing dynamic fragmentation. By contrast, flows dominated by graininertial stresses $\left(\delta_{\mathrm{s}} / \delta_{1}>0.26\right)$ exhibit up to $66 \%$ more impulses and 4.3 times more fragmentation. Dynamic fragmentation of bi-disperse flows impacting a rigid barrier can dissipate about $30 \%$ of the total flow energy.
\end{abstract}

Key words: Bi-dispersity; dynamic fragmentation; impact; rigid barrier; centrifuge modelling 


\section{Introduction}

Geophysical flows are stress-dependent phenomena, which surge downslope at high velocities and rapidly evolve from triggering to deposition (Cui et al. 2017). These flows consist of particles that cover a wide range of sizes, ranging from gravel to boulder. Bi-dispersity, two different particle sizes, is a prerequisite for grain-size segregation (Gray and Chugunov 2006), which transports large particles to the free surface and accumulates them at the flow front (Calvetti et al. 2000; Thornton et al. 2006). Grain-size segregation has been investigated in the context of the transportation (Zhou and Ng 2010) and deposition (Johnson et al. 2012) stages of mass-wasting processes. However, the effects of grain-size segregation on the impact forces exerted on rigid barriers (Lo 2000) remain a crucial scientific challenge. Large particles at the flow front can generate concentrated loads (Bowmen et al. 2010; Cui et al. 2018), which have been reported to severely damage reinforced-concrete structures situated in their flow paths (Zhang et al. 1996).

Aside from the concentrated loads that are exerted on rigid barriers, large particles can also fragment upon impact. The degree to which fragmentation occurs governs the resulting forces exerted on a barrier. One would expect that a particle that fragments would exhibit a smaller impact force compared to that of a particle that stays intact. Dynamic fragmentation is the reduction of the size of particles in rock avalanches and debris flows (Davies et al. 1999). This feature occurs when fast-moving particles grind or collide with each other, or with their boundaries (Bond 1952). Dynamic fragmentation has been evaluated against the runout distance for rock avalanches using centrifuge model tests (Bowman et al. 2012). These studies have proven that the centrifuge is a suitable tool for recreating the absolute stress state of a granular assembly (Schofield 1980; Taylor 1984; Bowman et al. 2010) and to generate stresses 
that enable the investigation of dynamic fragmentation (Imre et al. 2010), which would otherwise not be possible using small-scale experiments (Davies and McSaveney 1999).

Bowman et al. (2012) demonstrated that the mobility of a rock avalanche is related to the relative breakage $B_{\mathrm{R}}$ of the flow material, as proposed by Hardin (1985) and given as follows:

$B_{R}=\frac{B_{T}}{B_{P}}$

where $B_{\mathrm{T}}$ is the total breakage, and $B_{\mathrm{P}}$ is the breakage potential. Both parameters are obtained from the PSDs of the geo-material. Breakage potential $B_{\mathrm{P}}$ is defined as the area bounded by the PSD before fragmentation, while total breakage $B_{\mathrm{T}}$ is defined by the area bounded between the PSDs before and after fragmentation. Breakage potential indicates the maximum breakage of particles that can occur by fragmentation, while total breakage represents the breakage that actually occurred.

$\mathrm{Ng}$ et al. (2017) focused on the fundamental impact mechanisms of granular flow and viscous flow on a model rigid and flexible barriers ( $\mathrm{Ng}$ et al. 2016b) using the geotechnical centrifuge. In essence, a frictional sand material with a low degree of viscous shearing was compared to viscous fluid. Both of these flows were assumed to behave as a continuum with absence of large particles or inclusions. The study reported in Song et al. (2018) was a continuation of the previous studies to examine the effects of solid fraction on two-phase debris flows, sand and viscous fluid, impacting a rigid barrier. Furthermore, Song et al. (2018) examined the effects of particle size of dry granular flow impacting a rigid barrier. This study improved the characterisation between flow that behaves as a continuum and flow that behaves 
discretely during impact. These findings help to discern when a design should be continuumbased (hydrodynamic approach) or discrete based (Hertz equation).

Back-analyses of nine field cases by Locat et al. (2006) show that fragmentation energy represents about $20 \%$ of the initial potential energy of a geophysical flow. Furthermore, findings show that the effects of dynamic fragmentation are augmented at the front of geophysical flows where large and inertial particles collide with each other as they travel downslope.

Although studies have been carried out to investigate the effects of dynamic fragmentation during transportation, there remains a knowledge gap on the effects of dynamic fragmentation on the impact force exerted on a barrier. Significant dynamic fragmentation was observed in the centrifuge tests conducted by Song (2017) and Song et al. (2018). However, the effects of dynamic fragmentation on the impact force exerted on the model rigid barrier was not examined. Clearly, the contrasting effects of sharp impulses induced by large particles on a rigid barrier and the dynamic fragmentation of these particles, which dissipate energy, influence the resisting force required by a rigid barrier. These pertinent features are important in the design of a rigid barrier and warrant further investigation.

In this paper, a series of centrifuge experiments were carried out. The experiments were designed to study the effects of bi-dispersity and dynamic fragmentation on the impact force exerted on a model rigid barrier. The size of the small particles was varied, while both the size of the large particles and the volume ratio between small and large particles were held constant. The novelty of this study lies in focusing on the degree of dynamic fragmentation after granular flows impact a rigid barrier. The scientific challenges pertaining to what flow 
compositions are most prone to dynamic fragmentation and the loading response on a rigid barrier are investigated.

\section{Limitations of hydrodynamic impact model for rigid barriers}

The current deficiency in our understanding of the impact force exerted on barriers is reflected by international guidelines (VanDine 1996; Proske et al. 2011; Kwan 2012). Generally, an estimate of the impact forces of geophysical flows is assumed to be dominated by the dynamic forces. A generalised form of hydrodynamic equation (Hungr et al. 1984; WSL 2009; Kwan 2012; Bugnion et al. 2010; Bugnion et al. 2012), which assumes the momentum upon impact is destroyed, on a rigid barrier is given as follows:

$F=\alpha \rho v^{2} h w$

where $F$ is the impact force, $\alpha$ is the dynamic pressure coefficient, $\rho$ is the debris density, $v$ is the flow velocity, $h$ is the flow thickness and $w$ is the channel width. The main limitation of the hydrodynamic equation is that the flow is assumed as an equivalent fluid. This means that the effects of particle size and dynamic fragmentation are not explicitly considered. The assumption that momentum is completely destroyed upon impact in Eqn. 2 is not appropriate for discrete particles impacting a barrier. This is because the impact force will obviously be augmented when the particles rebound off of the barrier. Therefore $\alpha$ is used to empirically compensate for this uncertainty in the flow composition. The deficiency in the hydrodynamic approach in predicting the impact force when large boulders are included in the flow is best documented by Zhang (1993). In the Jiangjia Ravine of China, back-calculated $\alpha$ values from 70 measurements are ranged from 3 to 5 . The variability is attributed to the inclusion of entrained boulders in the flows. Volkwein (2014) further emphasized that $\alpha$ is dependent on 
the flow composition. More specifically, for granular debris flows (1900 to $2300 \mathrm{~kg} / \mathrm{m}^{3}$ ), the $\alpha$ value should be 2 while for fluid-like debris flows (1600 to $2000 \mathrm{~kg} / \mathrm{m}^{3}$ ) the $\alpha$ value ranges from 0.7 to 1.0 . The higher solid fractions in granular debris flows entail higher dynamic pressure coefficients compared to fluid-like flows with lower solid fractions. The large particles are also likely to rebound upon impacting a rigid structure, and therefore augmenting the impact force (Jiang and Towhata 2013). Evidently, recommendations for $\alpha$ value are inconsistent and physical data is urgently required to elucidate the effects of particle size.

To capture the impact of boulders or a large and hard inclusion, the Hertz equation is often used to estimate the impact force. A modified version of the Hertz equation for a granite boulder impacting a reinforced concrete wall (Kwan 2012) is given as follows:

$F=K_{c} 4000 v^{1.2} R^{2}$

where $K_{\mathrm{c}}$ is the load reduction factor and $R$ is the boulder radius. The load-reduction factor $K_{\mathrm{c}}$ is empirically recommended as 0.1 . This value is for a perfectly elastic impact scenario, which is reduced by $90 \%$ to account for a plastic deformation during impact. The validity of the Hertz equation when more than a single particle impact a rigid barrier is a crucial scientific challenge that needs to be addressed.

\section{Centrifuge modelling}

The geotechnical centrifuge at The Hong Kong University of Science and Technology (Ng 2014) was used to carry out the experiments in this study. The centrifuge has a diameter of 8.4 $\mathrm{m}$ and a payload capacity of 400 g-ton. The tests were carried out at a g-level of 22.4. Details of the model setup, instrumentation, test programme and test procedures are described below. 


\section{Scaling and flow characterisation}

Details of scaling principles are discussed in Chikatamarla et al. (2006). The downslope motion of a geophysical flow is driven by gravitational potential, therefore the velocity scale $v$ is characterised using $(g L)^{1 / 2}$ (Iverson 1997; Valentino et al. 2008), where $L$ is the characteristic flow length. In centrifuge modelling, as the gravitational acceleration increases $N$ times, both the linear scale and inertial time scale are reduce by $N$ times, thereby resulting in a scale factor of unity for the flow velocity. Based on the fundamental principles of centrifuge modelling, the impact pressure $\left(P \sim \rho v^{2}\right)$ is the same as that of the prototype. The impact force $\left(F \sim \rho v^{2} A\right)$ induced on the barrier has a scale factor of $1 / N^{2}$, where $A$ is the impact area on the barrier $(\mathrm{Ng}$ et al. 2017). According to Hertz contact theory, the boulder impact force $F$ also has a scale factor of $1 / N^{2}$. A summary of relevant scaling laws is given in Table 1 .

The collisional regime of the flow can be described by kinetic theory. The kinetic energy dissipates differently depending on collisions within the granular medium (Azanza et al. 1999). To quantify the collisions among grains within the flow, the relative importance of inertial and contact grain stresses can be assessed using the Savage Number (Savage 1984). This dimensionless group is used to characterise the mesoscopic stresses of the granular assembly and correlate with the degree of dynamic fragmentation and the force exerted on the rigid barrier. The Savage Number is given as follows:

$$
N_{\text {Sav }}=\frac{\delta^{2} \dot{\gamma}^{2}}{g h}
$$

where $\delta$ is the grain diameter and $\dot{\gamma}$ is the shear strain rate. The Savage Number can characterise whether flows are frictional or contact-dominated. 
$\dot{\gamma}=\frac{v_{s}-v_{b}}{h}$

where $v_{\mathrm{s}}$ is the flow velocity at the surface and $\mathrm{v}_{\mathrm{b}}$ is flow velocity at the base. The empirical threshold between the two regimes is reported as $N_{\mathrm{Sav}}=0.1$ (Savage and Hutter 1989). A summary of the parameters used to calculate $N_{\text {Sav }}$ is given in Table 2.

\section{Model setup and instrumentation}

Figure 1 shows the model setup used in this study. A side view of the model container, with dimensions of $1245 \mathrm{~mm}$ in length, $350 \mathrm{~mm}$ in width, and $850 \mathrm{~mm}$ in depth, on the centrifuge platform is shown in Fig. 1a. This container has a Perspex window for capturing the flow kinematics of the tests. A model slope with an incline of $25^{\circ}$ was installed in the model container. A storage container with a volume of $0.03 \mathrm{~m}^{3}$ was mounted above the upstream end of the model slope. A hinged-door at the bottom of the storage container was used to retain the source material. This door was controlled using a hydraulic actuator to release the source material on to the channel in-flight.

A magnesium alloy plate, $200 \mathrm{~mm}$ in height and $233 \mathrm{~mm}$ in width, was installed perpendicularly to the channel to model a rigid barrier (Fig. 1b). The Young's modulus of the magnesium alloy plate is the upper limit of that of reinforced concrete. A through-hole compression load cell was installed between an alloy plate and aluminum buttress to measure the total load on the model rigid barrier. A sampling rate of $20 \mathrm{kHz}$ was selected to ensure the details of the dynamic process were captured. As a single load cell was used to measure the total load acting on the barrier. This means that spikes are likely occurring at different locations along the barrier. Since the barrier is not cantilevered and meant to measure load one-dimensionally (slope parallel direction), details pertaining to the load distribution and 
implications on the induced bending moment could not be interpreted. A high-speed camera, with a sampling rate of 640 frames per second at a resolution of $1300 \times 1600$ pixels was used to record the impact kinematics. High-speed images enabled particle imagery velocimetry (PIV) for analysing the velocity fields during impact (White et al. 2003).

\section{Test programme}

To investigate the effects of bi-dispersity and dynamic fragmentation on the impact force exerted on a model rigid barrier, both control and impact tests were carried out. Prior to impact tests, control tests, without a barrier, were used to characterise the flow dynamics.

The model size of the small particles forming the bi-disperse flows, was varied as $3 \mathrm{~mm}$ (67 $\mathrm{mm}$ in prototype), $10 \mathrm{~mm}$ (224 $\mathrm{mm}$ in prototype) and $22 \mathrm{~mm}$ (462 $\mathrm{mm}$ in prototype) while the size of the large particles remained unchanged as $39 \mathrm{~mm}$ (874 $\mathrm{mm}$ in prototype) (Fig. 2). A summary of the test programme is given in Table 2 . The volume fraction of large particle (39 $\mathrm{mm}$ ) to small particles (22 mm, $10 \mathrm{~mm}$ and $3 \mathrm{~mm}$ ) was selected as $3: 7$. Casagli et al. (2003) summarised 42 granular flows from field study. The boulder inclusion of the granular flows was ranged from $10 \%$ to $60 \%$ of the total grains. High volume fraction of large particles would dominate the effects of bi-dispersity on dynamic fragmentation. After consideration of achieving research purposes, $30 \%$ of large particles was selected to model bi-disperse flows in this study. $0.874 \mathrm{~mm}, 462 \mathrm{~mm}, 224 \mathrm{~mm}$ and $67 \mathrm{~mm}$ grains are classified as large boulders, boulders, cobbles and coarse gravels (Bell 2013). To characterise the PSDs of these bi-disperse flows, the diameter of the small particles was normalised by the diameter of the large particles $\left(\delta_{\mathrm{s}} / \delta_{1}\right)$. To some extent, the large particles represent the boulders that are often entrained and transported to the front of a flow. A $\delta_{\mathrm{s}} / \delta_{1}$ value of zero represents mono-disperse flows comprising only large particles, whereas a $\delta_{\mathrm{s}} / \delta_{1}$ of unity represents mono-disperse flows 
comprising particles of the same size. For a constant volume ratio between the small and large particles, a vanishing $\delta_{\mathrm{s}} / \delta_{1}$ can be expected that the flow to be dominated by contact stresses. By contrast, for a $\delta_{\mathrm{s}} / \delta_{1}$ of unity, one can expect the flow to be dominated by inertial stresses. The impact force exerted by flows that are highly inertial tends to induce sharp impulses, whereas the impact force exerted by flows that are highly contact-dominated tends to dissipate flow kinetic energy rapidly and does not exhibit sharp impulses (Song et al. 2018).

\section{Test procedures}

A model volume of $0.027 \mathrm{~m}^{3}$ of source material was prepared in the storage container, at 22.4 $\mathrm{g}$ this volume is equivalent to $170 \mathrm{~m}^{3}$ in prototype. The samples were initially prepared to reach a target bulk density of $1300 \mathrm{~kg} / \mathrm{m}^{3}$ by firstly layering the large particles near the base of the container, followed by the small particles. The centrifuge was spun to the target g-level of 22.4. Afterwards, the hinged-door was released by activating the hydraulic actuator used to secure the door. The source material was mobilised on to the slope and flowed into the model rigid barrier. Simultaneously, the data logger and high-speed cameras were obtained measurements. After each test, the glass fragments inside the centrifuge model container were collected. The fragments were then sorted using sieve analysis and weighed using an electronic balance. The size and mass of fragmented glass spheres were recorded to quantify the degree of dynamic fragmentation. PSDs before and after each test were obtained.

\section{Interpretation of test results}

\section{Observed impact kinematics}

The impact kinematics are shown for bi-disperse flows with normalised particle diameters of $\delta_{\mathrm{s}} / \delta_{1}=0.08$ and 0.56 in Figs. 3 and 4, respectively. The difference between these two flows lies in the size of the small particles comprising the flow. The volume ratio between the small and 
large particles remains constant as 7:3. Snapshots from the high-speed camera are shown on the left and the corresponding PIV analysis is shown on the right. The impact time is normalised as unity $(t=1.00 \mathrm{~s})$ just as the flow front reaches the model rigid barrier.

Visualisations were made based on high-speed imagery and using PIV analysis (White et al. 2003). Traces of glass fragments in high-speed images were observed visually. The collisional processes were quite chaotic and the corresponding PIV vectors do not behave in a systematic manner. Figure 3 shows the impact kinematics of the bi-disperse flow with a normalised particle diameter of $\delta_{\mathrm{s}} / \delta_{1}=0.08$. The flow front impacts the barrier at $t=1.00 \mathrm{~s}$ (Fig. 3a). At $t=1.67 \mathrm{~s}$, dynamic fragmentation of the large particles is observed and the small particles runup along the vertical face of the model rigid barrier (Fig. 3b). This runup is corroborated by the vertical vectors observed in the corresponding PIV analysis. Also, the accumulation of the large particles at the front and free surface of the flow, via kinetic sieving, is observed. At $t=2.43 \mathrm{~s}$, the small particles cascade over the barrier and deposition forms at the barrier. High-speed images showed deposited material in front of the barrier, which was corroborated with near static vectors from PIV. Vectors near the top of the barrier correspond with particles cascading over the barrier (Fig. 3c). At $t=3.02 \mathrm{~s}$, the size of the deposited material at the base has increased and only a thin layer of granular material flows over the surface of the deposited material (Fig. 3d).

Observations were also made based on high-speed imagery and PIV. Figure 4 shows the impact kinematics of the flow with a normalised particle diameter of $\delta_{\mathrm{s}} / \delta_{1}=0.56$. Similar to the impact kinematics observed for $\delta_{\mathrm{s}} / \delta_{1}=0.08$, the flow front approaches and impacts the barrier at $t=1.00 \mathrm{~s}$ (Fig. 4a). A more inertial flow front, compared to $\delta_{\mathrm{s}} / \delta_{1}=0.08$, embedded with fragments is observed. More obvious fragmentation has occurred and is attributed to the 
collisional stresses of the large particles colliding at the front of the flow. The corresponding PIV vectors are less coherent as more dynamic fragmentation occurs. After the flow impacts the barrier at $t=1.67 \mathrm{~s}$ (Fig. 4b), fragments are observed at the interface between the flow and the barrier. At $t=2.34 \mathrm{~s}$, more fragments are observed at the interface between the flow and the barrier (Fig. 4c). Simultaneously, the deposited material is observed at the base of the barrier. The collisional nature of the flow remains obvious near the free-surface and no significant overflow is observed. At $t=3.02 \mathrm{~s}$ (Fig. 4d), the impact process begins to reach static condition and only a thin layer of flow is observed on top of the deposited material.

\section{Influence of particle size}

Figure 5 show a comparison of the force time-histories for bi-disperse flows with normalised particle diameters of $\delta_{\mathrm{s}} / \delta_{1}=0.08$ (Fig. 5a), 0.26 (Fig. 5b), and 0.56 (Fig. 5c). The dynamic loads are normalised with the static load for each test. The time is unity $(t=1.00 \mathrm{~s})$ when the flow front reaches the model rigid barrier. The estimated load using the hydrodynamic equation (Eqn. 2) with an $\alpha$ value of 2.5 (Kwan 2012) and the Hertz equation (Eqn. 3) with a $K_{\mathrm{c}}$ value of 0.1 (Kwan 2012), and the superposition of Eqns. 2 and 3 are shown for reference.

For $\delta_{\mathrm{s}} / \delta_{1}=0.08$, numerous sharp impulses are generated when the flow impacts the rigid barrier (Fig. 5a). The peak impulse is greater than the load estimated using the Hertz equation and the hydrodynamic equation by more than five times and $13 \%$, respectively. The impulses eventually diminish as the impact process reaches a static state. The superposition of both Eqns. 2 and 3 successfully bounds the measured peak load. To highlight the uniqueness of the impact behaviour of bi-disperse flows, the impact behaviour of dry Leighton Buzzard (LB) Fraction C sand and a two-phase mixture tests, with 50\% solid fraction of LB Fraction C sand (Song et al. 2018), are shown for comparison. Dry LB Fraction C sand exhibits a continuous and 
progressive loading pattern towards a static state without an obvious dynamic peak or sharp impulses. This observed behaviour is similar to that of the loading pattern reported for lowdischarge granular flow tests by Ashwood and Hungr (2016). Also, the tapered shape of the flow front is instrumental to the development of the progressive loading behaviour. The twophase mixture, however, exhibits a distinct peak load before a static state. The two-phase mixture is fluidised so that it takes a longer time to reach a static condition. The dry sand and two-phase mixture tests conducted by Ng et al. (2017) and Song et al. (2018) are bounded by the superposition of both Eqns. 2 and 3. However, the bi-disperse flows with hard and large inclusions cannot be bounded by existing guidelines. These results suggest that the entrainment of large and hard inclusions in a geophysical flow warrants a careful consideration in the design of rigid barriers.

Similarly, for bi-disperse flow with a normalised particle diameter of $\delta_{\mathrm{s}} / \delta_{1}=0.26$ (Fig. 5b), a multitude of sharp impulses are generated upon impact. These impulses eventually diminish towards a static state. However, the peak impulse exceeds the superposition of Eqns. 2 and 3 by about $9 \%$. Results show that the number of impulses generated for bi-disperse flows with normalised particle diameter $\delta_{\mathrm{s}} / \delta_{1}=0.26$ are greater than that for normalised particle diameter of $\delta_{\mathrm{s}} / \delta_{1}=0.08$. The more effective attenuation of impulses for $\delta_{\mathrm{s}} / \delta_{1}=0.08$ is due to more enduring contacts between the small particles, which are more effective at dissipating energy through friction. Similarly, for the bi-disperse flow with normalised particle diameter $\delta_{\mathrm{s}} / \delta_{1}=0.56$ (Fig. 5c), similar sharp impulses are generated. However, there are significantly more impulses compared to that observed in Figs. 5a and 5b. More specifically, 66\% more impulses are counted when the normalised particle diameter $\delta_{\mathrm{s}} / \delta_{1}$ is increased from 0.08 to 0.56 . The impulses from $\delta_{\mathrm{s}} / \delta_{1}=0.56$ also exceed the superposition of Eqns. 2 and 3 by as much as $48 \%$. Clearly, the inclusion of large particles and the relative size of the finer particles strongly 
influence the impact force on a rigid barrier and the use of existing guidelines warrants careful consideration.

A comparison of the force time-histories shows that as $\delta_{\mathrm{s}} / \delta_{1}$ of the bi-disperse flows is reduced, the flows are more effective at attenuating sharp impulses induced on the rigid barrier. This observation implies that small particles in a bi-disperse mixture play a significant role in cushioning the dispersive stresses of the large particles. A comparison with existing impact models corroborates that the Hertz equation, which assumes a single sphere impacting a flat surface cannot holistically predict the impact of a cluster of large particles impacting a rigid barrier. The hydrodynamic equation also does not appear to consistently be able to sufficiently bound the sharp impulses from the bi-disperse mixture in this study (Figs. 5b and 5c), even with a dynamic pressure coefficient of 2.5. This indicates that the discrete nature of flows with large particles tends to generate impulse that cannot be captured by the concept of an equivalent fluid (Hungr 1995) as implicitly assumed in Eqn. 2.

The results show the peak loads exerting on rigid barrier with the presence of discrete boulders within the flow. The superposition of the hydrodynamic equation and Hertz equation (Kwan 2012) is not sufficient to bound the measured impact loads in this study (Fig. 5). This implies that the existence of entrained boulders may require either the dynamic pressure coefficient $\alpha$ or the load reduction factor $K_{\mathrm{c}}$ to be modified to ensure a robust barrier design.

\section{Influence of grain stresses on impact force}

The influence of grain stresses, manifested in the $N_{\mathrm{Sav}}$ on the back-calculated $\alpha$ is shown in Fig. 6. The $\alpha$ values are back-calculated using the peak loads measured from each test. Reference lines are provided to indicate $\alpha$ values from different literatures. More particularly, $\alpha$ values 
are recommended by Zhang (1993) and Volkwein (2014). The $N_{\mathrm{Sav}}$ itself relies on a characteristic diameter, which is taken as the mean particle size based on the volume fraction in this study. Results show that as the flow becomes more inertial, or as the particle size ratio increases, $\alpha$ also increases. Results indicate that the hydrodynamic approach, which relies on a single $\alpha$ value, is not appropriate for capturing the effects of particle size for rigid barrier. The recommended value of $\alpha$ as proposed by Zhang (1993) can capture the short duration impulses exerted by large particles in bi-disperse flows. This is likely due to the prevalence of large boulders reported in the Jiangjia Ravine, which is reflected in the back-calculated $\alpha$ values. Findings also suggest that recommended $\alpha$ values are likely dependent on the prevailing geological settings and whether large clasts were present.

\section{Potential implications to barrier design}

The results in this study show that the recommended dynamic pressure coefficient in current design guidelines (Kwan 2012) is not sufficient to capture flows with large and hard inclusions. Evidently, a larger dynamic pressure coefficient is required (at least 5 for particle diameters up to $874 \mathrm{~mm}$ based on the results of this study). A cluster of large particles possess extremely high momentum, thereby resulting in high impact loads. To account for such induced loads, comprehensive boulder surveys are necessary for mitigation strategies before designing barriers. Also, installation of cushioning material is clearly an important feature for rigid barriers. Cushioning materials should no longer be prescriptive since they have been reported to attenuate impact loads by up to $89 \%$ ( $\mathrm{Ng}$ et al. 2016a; Lam et al. 2018).

\section{Dynamic fragmentation}

Dynamic fragmentation occurs when grains grind, crush and collide with each other (Bond 1952). In this study, dynamic fragmentation may occur during any of the three stages. First, 
when the flow is released from the storage container, dynamic fragmentation may occur as the flow impacts at the base of the channel. Second, as particles experience crushing, grinding, and collisions before the flow impacts the model rigid barrier. Third, during impact against the model rigid barrier. A summary of the fragmentation measurements is given in Table 3 . The percentage of dynamic fragmentation for small particles in the bi-disperse flows is from $0.2 \%$ to $1.1 \%$, while dynamic fragmentation for large particles is from $3.5 \%$ to $12.5 \%$. Measurements indicate that fragmentation predominantly occurs for large particles, which are more inertial (Fig. 6).

Figure 7 shows PSDs before and after each test for bi-disperse flows with normalised particle diameters of $\delta_{\mathrm{s}} / \delta_{1}=0.08,0.26$, and 0.56 . To study the effects of fragmentation that occurs when the granular material impacts the channel, relevant tests on a flexible barrier were repeated. The purpose of the flexible barrier is to quantify the effect of dynamic fragmentation resulting from impact. Song et al. (2018) reported that flexible barriers are very effective at attenuated sharp impulses and thus the dynamic fragmentation during impact. These flexible barrier tests serve merely as a reference in an effort to isolate the effects of dynamic fragmentation during impact upon barriers. Although not the focus of this study, details of the flexible barrier are discussed in $\mathrm{Ng}$ et al. (2016b).

Figure 7a shows a comparison of the PSDs for the bi-dispersed flow with a normalised particle diameter $\delta_{\mathrm{s}} / \delta_{1}=0.08$ before and after the test for barriers. Results indicate that dynamic fragmentation is predominantly from the large particles. This is because the small particles act as a cushioning medium for the larger particles. The cushioning medium effectively attenuates flow kinetic energy through enduring frictional-contact stresses. The cushioning effect results in less impulses than that of the flows with larger particle diameter ratios. Figure $7 \mathrm{~b}$ shows a 
comparison of the PSDs for the bi-disperse flow with a normalised particle diameter of $\delta_{\mathrm{s}} / \delta_{1}=$ 0.26 before and after the test for barriers. Results show a more dynamic fragmentation of both the small and large particles compared to that in Fig. 7a, where the size of the small particles is about three times smaller than that presented in Fig. 7b. The higher degree of dynamic fragmentation is caused by the increase in particle diameter of the small particles comprising the bi-disperse flows. The less effective cushioning medium increase the degree of dynamic fragmentation of the large particles. Figure $7 \mathrm{c}$ shows a comparison of the PSDs for the bidisperse flow with a normalised particle diameter of $\delta_{\mathrm{s}} / \delta_{1}=0.56$ before and after the test for barriers. The degree of dynamic fragmentation reveals a much wider range of fragment size than both Figs. 7a and $7 \mathrm{~b}$. The more inertial the flow is, the higher the resulting impact force exerted on a rigid barrier, and therefore a higher degree of dynamic fragmentation. The relative breakage is calculated increased in about 4 times when normalised particle diameter $\delta_{\mathrm{s}} / \delta_{1}$ is increased from 0.08 to 0.56 .

Comparisons of the PSDs are also shown for the bi-dispersed flow after the test for a model flexible barrier in each figure. Similar to tests of rigid barrier, more dynamic fragmentation is found with increase in normalised particle diameter $\delta_{\mathrm{s}} / \delta_{1}$. Effects of bidispersity can also be observed for tests for tests with flexible barrier. However, by comparing the same normalised particle diameter, the corresponding relative breakage is greatly reduced. The reduction in relative breakage is due to the absence of dynamic fragmentation during impact on a highly-deformable flexible barrier. The influence from distinguished barriers is further discussed in Figure 8.

Figure 8 shows the effects of grain stresses, manifested in the $N_{\text {Sav }}$, on the degree of dynamic fragmentation using relative breakage $B_{\mathrm{R}}$ (Eqn. 1) as a benchmark. Relative breakage 
$B_{\mathrm{R}}$, representing degree of dynamic fragmentation increases with $N_{\text {Sav }}$. Although, dynamic fragmentation clearly occurred as the flow impacts the rigid barrier, there were also fragments embedded in the flow before impacting the model rigid barrier. A more systematic investigation is required to consider the stage at which the particles begin to fragment. Notwithstanding, this study shows that dynamic fragmentation from impact is a very significant contributor to the dissipation of total energy. Results show that the grain stresses in the flow, specifically $N_{\text {Sav }}$, is directly related to relative breakage $B_{\mathrm{R}}$. Higher grain inertial stress, and therefore higher $N_{\mathrm{Sav}}$, results in a higher degree of dynamic fragmentation. The measured relative breakage for a model flexible barrier is reduced up to $74 \%$ for normalised particle diameter $\delta_{\mathrm{s}} / \delta_{1}$ of 0.26 compared to that measured for a model rigid barrier.

Two references are shown for rock avalanches in Ashburton, New Zealand (McSaveney et al. 2000) and in La Madeleine, France (Locat et al. 2006). One would expect that the degree of dynamic fragmentation should be more significant for rock avalanches undergoing long transportation distances compared to the shorter transportation distances travelled by the model flows in this study. However, the bi-disperse flows with a normalised particle diameter of $\delta_{\mathrm{s}} / \delta_{1}$ $=0.56$ reveals a relative breakage of about 0.28 . This value suggests that despite the short transportation distance in the centrifuge test, the degree of dynamic fragmentation predominantly resulted from either the impact of flow material onto the channel base or from impact on the model rigid barrier.

Figure 9 shows a comparison of the estimated fragmentation energy $E_{\mathrm{F}}$ with relative breakage $B_{\mathrm{R}}$ in this study. The fragmentation energy $E_{\mathrm{F}}$ of the bi-disperse flows is calculated by considering the potential energy, kinetic energy and frictional energy of the flow, as characterised by Heim (1932). Based on the interpretation of nine field rock avalanches, about 
$20 \%$ of the potential energy is converted to fragmentation energy (Locat et al. 2006). Findings also suggest that despite the short transportation length in this study, the degree of dynamic fragmentation for bi-disperse flows with normalised particle diameters of $\delta_{\mathrm{s}} / \delta_{1}=0.26$ and $\delta_{\mathrm{s}} / \delta_{1}$ $=0.56$, exhibit more than $20 \%$ in energy dissipation via fragmentation. The calculated fragmentation energy $E_{\mathrm{F}}$ of $\delta_{\mathrm{s}} / \delta_{1}=0.56$ is even up to $31 \%$. This indicates that dynamic fragmentation from impact may be more significant compared to dynamic fragmentation that occurs during transportation. Notwithstanding, a comparison between Figs. 6 and 8 support that the cushioning effect resulting from bi-dispersity is more effective at attenuating sharp impulses compared to dynamic fragmentation during impact. The back-calculated fragmentation energy $E_{\mathrm{F}}$ from flows impacting a model flexible barrier shows a decrease in relative breakage up to $49 \%$ for normalised particle diameter $\delta_{\mathrm{s}} / \delta_{1}=0.26$. The flexibility of the barrier is very effective at attenuating the impact force and therefore reduces dynamic fragmentation upon impact. Correspondingly, the fragmentation energy is also reduced.

\section{Conclusions}

A series of centrifuge tests were carried out to investigate the effects of bi-dispersity and dynamic fragmentation on the impact force exerted on a model rigid barrier. The ratio of small to large particles, and the diameter of the large particles were held constant, while the diameter of small particles was varied. Findings from this study are drawn as follows:

a) Normalised particle diameter $\delta_{\mathrm{s}} / \delta_{1}$ governs the collisional regime of the bi-disperse flows. Bi-dispersity determines if the flow is dominated by grain-contact or graininertial stresses and is characterised by Savage Number.

b) An $\delta_{\mathrm{s}} / \delta_{1}$ tending towards unity characterises inertial flows that exerts sharp impulses, and a diminishing $\delta_{\mathrm{s}} / \delta_{1}$ characterises the progressive attenuation of these sharp impulses 
by the small particles. Bi-disperse flows dominated by grain-contact stresses $\left(\delta_{\mathrm{s}} / \delta_{1}<\right.$ 0.26) develop a cushioning effect to attenuate the inertia of large particles. Dynamic load upon a barrier is highly reduced upon and results in less dynamic fragmentation and impulses. On the contrary, bi-disperse flows dominated by grain-inertial stresses $\left(\delta_{\mathrm{s}} / \delta_{1}>0.26\right)$ enhance both impulses and dynamic fragmentation.

c) The bi-disperse flows in this study, specifically with $\delta_{\mathrm{s}} / \delta_{1}=0.26$ and $\delta_{\mathrm{s}} / \delta_{1}=0.56$, exceed the superposition of both the Hertz and hydrodynamic equation. This is because of the assumption in the hydrodynamic equation that momentum is completely destroyed upon impact. In reality, large particles will rebound and augment the resulting resisting force by the barrier. This is corroborated by back-calculated $\alpha$ values where an abundance of boulders were considered. This suggests that current impact models cannot capture the effects of a cluster of large particles impacting a rigid barrier. Results show that a dynamic pressure coefficient up to 5 is required to capture the sharp impulse loads induced by a cluster of large particles with diameters of up to $874 \mathrm{~mm}$. Furthermore, cushioning layers are recommended for providing a practical alternative for optimising barrier designs.

d) Dynamic fragmentation bi-disperse flows impacting a rigid barrier can be as high as $31 \%$ of the total flow energy. This suggests that fragmentation energy is enhanced due to the impact against a rigid barrier than a flexible barrier. It plays a significant role in the energy dissipation process. Test results corroborate that dynamic fragmentation is an important mechanism to consider. Considering the effects of grinding, collisions, and impact with its boundary on dynamic fragmentation would be valuable to enhancing our understanding on the impact behaviour between geophysical flows and barriers.

e) This study fundamentally studies the effects of impact load exerted on rigid structure by both flow composition and quantities the degree of dynamic fragmentation of bidisperse flows after intense impact. However, this conclusion is limited to apply in 
practical engineering as it is technically difficult to quantify both flow composition and dynamic fragmentation of a granular flow. More research is warranted to establish an engineering approach to consider both PSD and boulders in field. 


\section{Acknowledgements}

The authors are grateful for financial support from the theme-based research grant T22603/15N and the general research fund 16209717 provided by the Research Grants Council of the Government of Hong Kong SAR, China. The authors are grateful for the financial sponsorship from the National Natural Science Foundation of China (51709052) and the support of the HKUST Jockey Club Institute for Advanced Study. The authors are grateful for the support of the HKUST Jockey Club Institute for Advanced Study and the financial support by the Hong Kong Jockey Club Disaster Preparedness and Response Institute (HKJCDPRI18EG01). 


\section{References}

Azanza, E., Chevoir, F., and Moucheront, P. 1999. Experimental study of collisional granular flows down an inclined plane. Journal of Fluid Mechanics, 400: 199-227.

Ashwood, W., and Hungr, O. 2016. Estimating total resisting force in flexible barrier impacted by a granular avalanche using physical and numerical modeling. Canadian Geotechnical Journal, 53(10): 1700-1717.

Bell, F.G. 2013. Engineering properties of soils and rocks. Butterworth-Heinemann Ltd., Oxford, UK.

Bond, F.C. 1952. The third theory of comminution. Mining Engineering, 4: 484.

Bowman, E.T., Laue, J., Imre, B., and Springman, S.M. 2010. Experimental modelling of debris flow behavior using a geotechnical centrifuge, Canadian Geotechnical Journal, 47(7): 742-762.

Bowman, E.T., Take, W.A., Rait, K.L., and Hann, C. 2012. Physical models of rock avalanche spreading behavior with dynamic fragmentation, Canadian Geotechnical Journal, 49(4): 460-476.

Bugnion, L., McArdell, B.W., Bartelt, P., and Wendeler, C. 2012. Measurements of hillslope debris flow impact pressure on obstacles. Landslides, 9(2): 179-187.

Bugnion, L., and Wendeler, C. 2010. Shallow landslide full-scale experiments in combination with testing of a flexible barrier. WIT Transactions on Engineering Sciences, 67: 161173.

Calvetti, F., Crosta, G., and Tatarella, M. 2000. Numerical simulation of dry granular flows: from the reproduction of small-scale experiments to the prediction of rock avalanches. Rivista Italiana di Geotecnica, 2(2000): 21-38.

Casagli, N., Ermini, L., and Rosati, G. 2003. Determining grain size distribution of the material composing landslide dams in the Northern Apennines: sampling and 
processing methods. Engineering geology, 69(1-2): 83-97.

Chikatamarla, R., Laue, J., and Springman, S.M. 2006. Centrifuge scaling laws for guided free fall events including rockfalls. International Journal of Physical Modelling in Geotechnics, 6(2): 15-26.

Choi, C.E., Ng, C.W.W., Au-yeung, S.C.H., and Goodwin, G.R. 2015. Froude characteristics of both dense granular and water flows in flume modelling. Landslides, 12(6): 11971206.

Cui, Y., Zhou, X., and Guo, C. 2017. Experimental study on the moving characteristics of fine grains in wide grading unconsolidated soil under heavy rainfall. Journal of Mountain Science, 14(3): 417-431.

Cui, Y., Choi, C.E., Liu, L.H.D., and Ng, C.W.W. 2018. Effects of particle size of monodispersed granular flows impacting a rigid barrier. Natural Hazards, 91(3): 1179_ 1201.

Davies, T.R., and McSaveney, M.J. 1999. Runout of dry granular avalanches. Canadian Geotechnical Journal, 36(2): 313-320.

Davies, T.R., McSaveney, M.J., and Hodgson, K.A. 1999. A fragmentation-spreading model for long-runout rock avalanches. Canadian Geotechnical Journal, 36(6): 1096-1110.

Gray, J.M.N.T., and Chugunov, V.A. 2006. Particle-size segregation and diffusive remixing in shallow granular avalanches. Journal of Fluid Mechanics, 569: 365-398.

Hardin, B.O. 1985. Crushing of soil particles. Journal of Geotechnical Engineering, 111(10): 1177-1192.

Heim, A. 1932. Bergsturz und Menschenleben. Translated in 1989 by N. Skermer as Landslides and human lives. Bi-Tech Publishers, Vancouver, B.C.

Hungr, O. 1995. A model for the runout analysis of rapid flow slides, debris flows, and avalanches. Canadian Geotechnical Journal, 32(4): 610-623. 
Hungr, O., Morgan, G.C., and Kellerhals, R. 1984. Quantitative analysis of debris torrent hazards for design of remedial measures. Canadian Geotechnical Journal, 21(4): 663677.

Iverson, R.M. 1997. The physics of debris flows. Review of geophysics, 35(3): 245-296.

Imre, B., Laue, J., and Springman, S.M. 2010. Fractal fragmentation of rocks within Sturzstroms: insights derived from physical experiments within the ETH geotechnical drum centrifuge. Granular Matter, 12(3): 267-285.

Jiang, Y. J., and Towhata, I. 2013. Experimental study of dry granular flow and impact behavior against a rigid retaining wall. Rock Mechanics and Rock Engineering, 46(4): $713-729$.

Johnson, C.G., Kokelaar, B.P., Iverson, R., Logan, M., LaHusen, R.G., and Gray, J.M.N.T. 2012. Grain-size segregation and levee formation in geophysical mass flows. Journal of Geophysical Research: Earth Surface, 117: F01032.

Kwan, J.S.H. 2012. Supplementary technical guidance on design of rigid debris resisting barriers. GEO Report No. 270, Geotechnical Engineering Office, HKSAR.

Lam, C., Kwan, J.S.H., Su, Y., Choi, C.E., and Ng, C.W.W. 2018. Performance of ethylenevinyl acetate foam as cushioning material for rigid debris-resisting barriers. Landslides, 15(9): 1779-1786.

Lo, D.O.K. 2000. Review of natural terrain landslide debris resisting barrier design. GEO Report No. 104, Geotechnical Engineering Office, HKSAR.

Locat, P., Couture, R., Leroueil, S., Locat, J., and Jaboyedoff, M. 2006. Fragmentation energy in rock avalanches. Canadian Geotechnical Journal, 43(8): 830-851.

McSaveney, M.J., Davies, T.R., and Hodgeson, K.A. 2000. A contrast in deposit style and process between large and small rock avalanches. In Proceedings of the 8th International Symposium on Landslides, 26-30 June 2000. Cardiff, UK, pp. 1053- 
1058.

Ng, C.W.W. 2014. The state-of-art centrifuge modelling of geotechnical problems at HKUST. Journal of Zhejiang University SCIENCE A, 15(1): 1-21.

Ng, C.W.W., Choi, C.E., Su, A.Y., Kwan, J.S.H., and Lam, C. 2016a. Large-scale successive boulder impacts on a rigid barrier shielded by gabions. Canadian Geotechnical Journal, 53(10): 1688-1699.

Ng, C.W.W., Song, D., Choi, C.E., Koo, R.C.H. and Kwan, J.S.H. 2016b. A novel flexible barrier for landslide impact in centrifuge. Géotechnique Letters, 6(1): 221-225.

Ng, C.W.W., Song, D., Choi, C.E., Liu, L.H.D., Kwan, J.S.H., Koo, R.C.H. and Pun, W.K. 2017. Impact mechanisms of granular and viscous flows on rigid and flexible barriers. Canadian Geotechnical Journal, 54(2): 188-206.

Proske, D., Suda, J., and Hübl, J. 2011. Debris flow impact estimation for breakers. Georisk, 5(2): 143-155.

Savage, S.B. 1984. The mechanics of rapid granular flows. Advanced in Applied Mechanics, 24: $289-366$.

Savage, S.B., and Hutter, K. 1989. The motion of a finite mass of granular material down a rough incline. Journal of Fluid Mechanics, 199: 177-215.

Schofield, A.N. 1980. Cambridge geotechnical centrifuge operations. Géotechnique, 30(3): $227-268$.

Song, D. 2017. Mechanisms of debris flow impact on rigid and flexible barriers. Ph.D. thesis, Department of Civil Engineering, The Hong Kong University of Science and Technology, Hong Kong, China.

Song, D., Choi, C.E., Zhou, G.G.D., Kwan, J.S.H., and Sze, H.Y. 2018. Impulse load characteristics of bouldery debris flow impact. Géotechnique Letters, 8(2): 111-117.

Taylor, R.N. 1984. Geotechnical centrifuge technology. Talor \& Francis, New York, USA. 
Thornton, A.R., Gray, J.M.N.T., and Hogg, A.J. 2006. A three-phase mixture theory for particle size segregation in shallow granular free-surface flows. Journal of Fluid Mechanics, 550: 1-25.

Valentino, R., Barla, G. and Montrasio, L. 2008. Experimental analysis and micromechanical modelling of dry granular flow and impacts in laboratory flume tests. Rock Mechanics and Rock Engineering, 41(1):153-177.

VanDine D.F. 1996. Debris flow control structures for forest engineering. Ministry of Forests, British Columbia, pp. 68.

Volkwein, A. 2014. Flexible debris flow barriers: Design and application. Swiss Federal Institute for Forest, Snow and Landscape Research, WSL.

White, D.J., Take, W.A., and Bolton, M.D. 2003. Soil deformation measurement using particle image velocimetry (PIV) and photogrammetry. Géotechnique, 53(7): 619-631.

WSL. 2009. Full-scale Testing and Dimensioning of flexible debris flow barriers. Technical report 1-22. WSL, Birmensdorf.

Zhang, S. 1993. A comprehensive approach to the observation and prevention of debris flows in China. Natural Hazards, 7(1): 1-23.

Zhang, S., Hungr, O., and Slaymaker, O. 1996. The calculation of impact force of boulders in debris flow. Debris flow observation and research. pp. 67-72.

Zhou, G.G., and Ng, C.W.W. 2010. Numerical investigation of reverse segregation in debris flow by DEM. Granular Matter, 12(5): 507-516. 


\section{Figure captions}

Fig. 1. Centrifuge model (all dimensions are in $\mathrm{mm}$ ): (a) side view of model setup on centrifuge platform; (b) side view of model rigid barrier

Fig. 2. Glass spheres used to model bi-disperse flows

Fig. 3. Side view of the observed kinematics and velocity fields from particle image velocimetry for bi-disperse flow $\left(\delta_{\mathrm{s}} / \delta_{1}=0.08\right.$ ): (a) $t=1.00 \mathrm{~s}$; (b) $t=1.67 \mathrm{~s}$; (c) $t=2.34 \mathrm{~s}$; (d) $t$ $=3.02 \mathrm{~s}$

Fig. 4. Side view of the observed kinematics and velocity fields from particle image velocimetry for bi-disperse flow $\left(\delta_{\mathrm{s}} / \delta_{1}=0.56\right.$ ): (a) $t=1.00 \mathrm{~s}$; (b) $t=1.67 \mathrm{~s}$; (c) $t=2.34 \mathrm{~s}$; (d) $t$ $=3.02 \mathrm{~s}$

Fig. 5. Normalised impact load time-history on rigid barrier: (a) $\delta_{\mathrm{s}} / \delta_{1}=0.08$; (b) $\delta_{\mathrm{s}} / \delta_{1}=0.26$; (c) $\delta_{\mathrm{s}} / \delta_{1}=0.56$

Fig. 6. Effects of grain-stresses on dynamic pressure coefficient for rigid barrier

Fig. 7. Measured particle size distributions (PSD) for rigid and flexible barrier: (a) $\delta_{\mathrm{s}} / \delta_{1}=$ $0.08 ;$ (b) $\delta_{\mathrm{s}} / \delta_{1}=0.26 ;$ (c) $\delta_{\mathrm{s}} / \delta_{1}=0.56$

Fig. 8. Influence between Savage Number $N_{\mathrm{Sav}}$ and relative breakage $B_{\mathrm{R}}$

Fig. 9. Relationship between fragmentation energy $E_{\mathrm{F}}$ and relative breakage $B_{\mathrm{R}}$ 

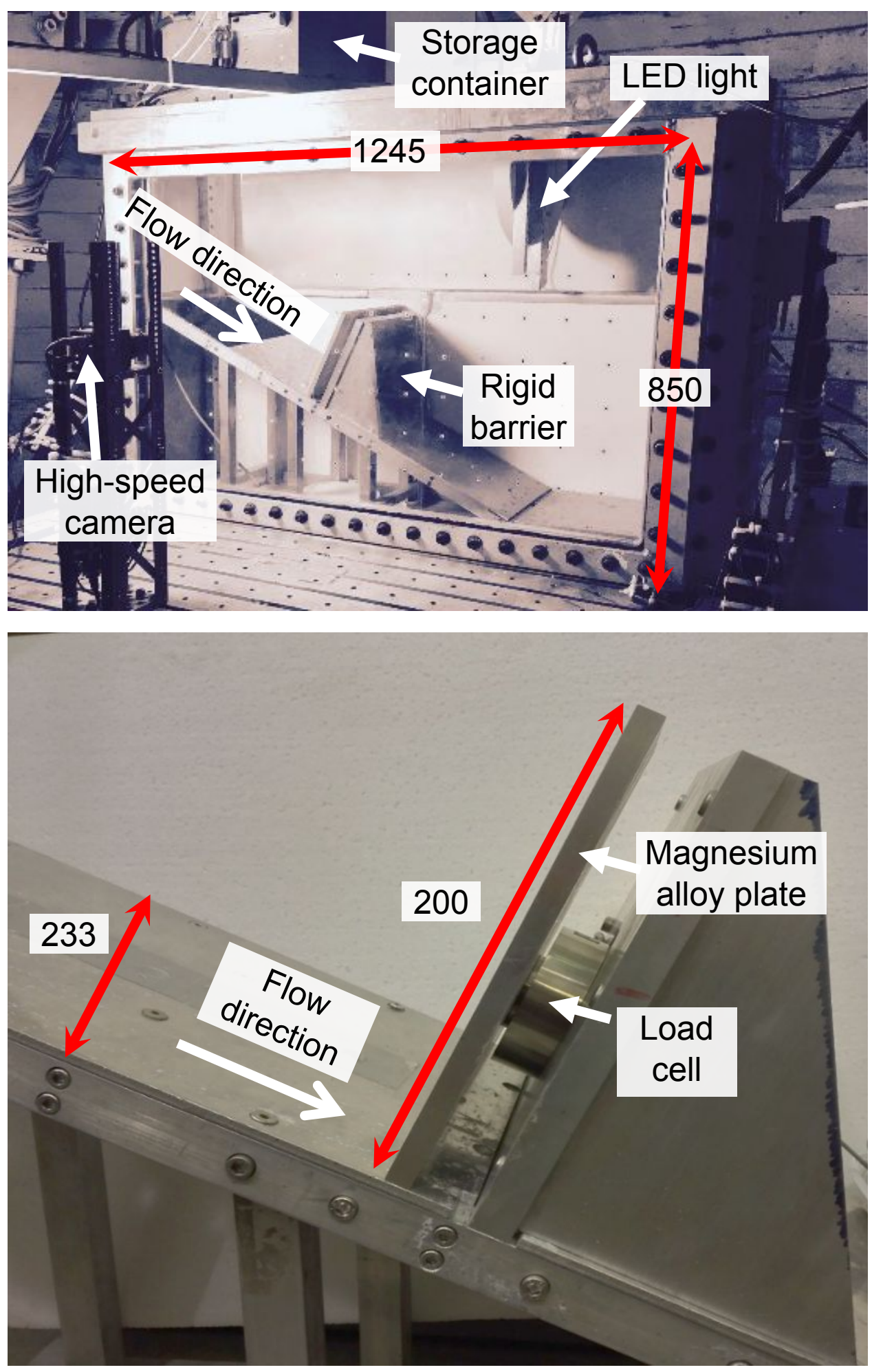

Fig. 1. Centrifuge model (all dimensions are in $\mathrm{mm}$ ): (a) side view of model setup on centrifuge platform; (b) side view of model rigid barrier 


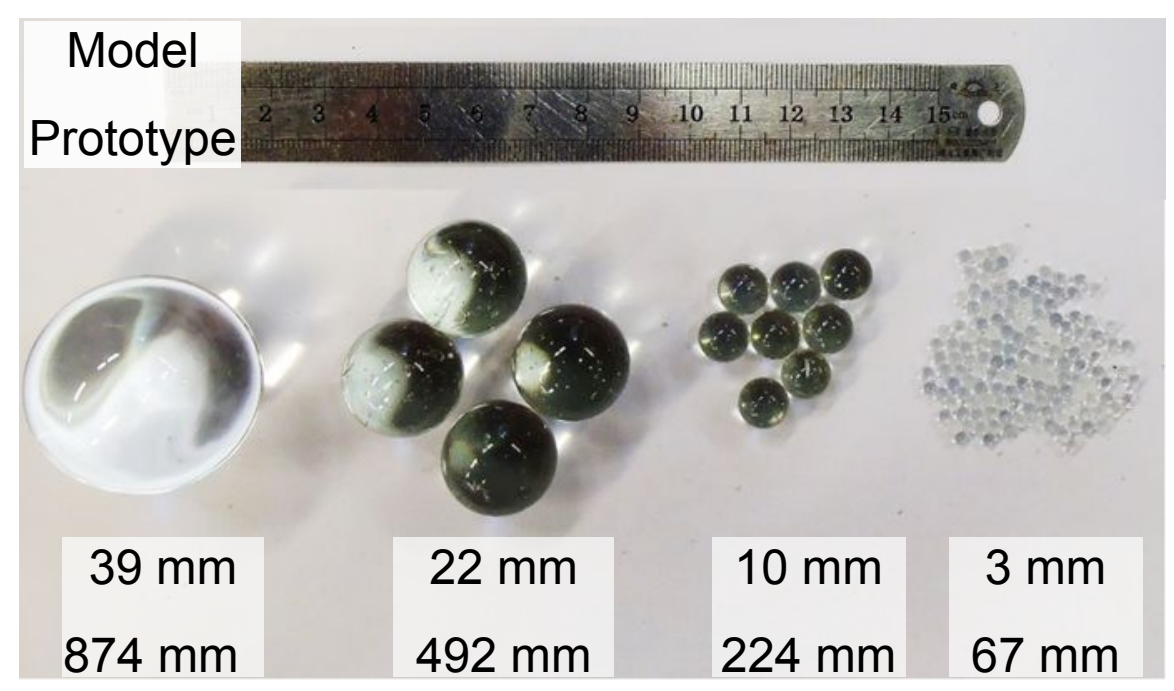

Fig. 2. Glass spheres used to model bi-disperse flows 


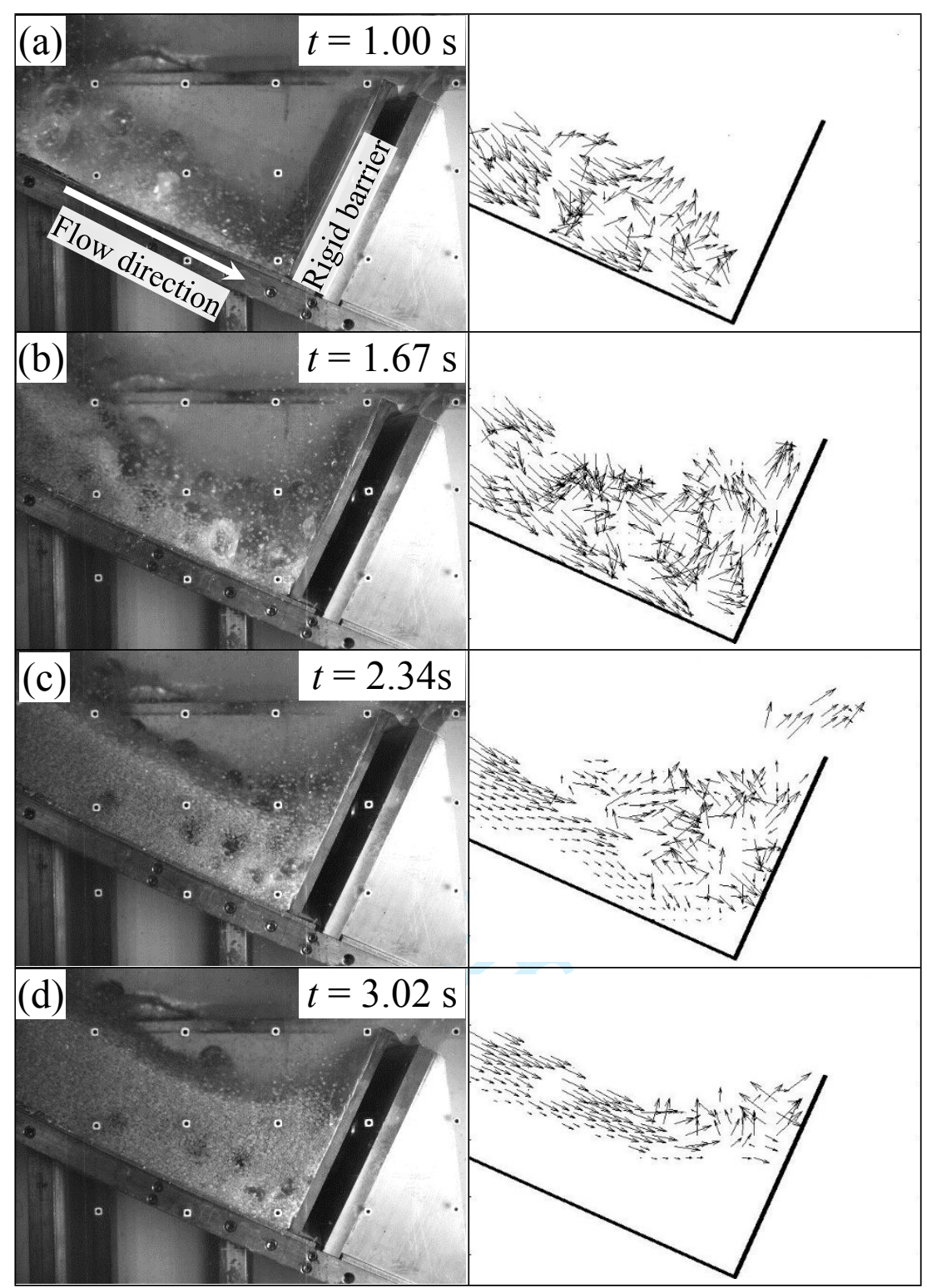

Fig. 3. Side view of the observed kinematics and velocity fields from particle image velocimetry for bi-disperse flow $\left(\delta_{\mathrm{s}} / \delta_{1}=0.08\right.$ ): (a) $t=1.00 \mathrm{~s}$; (b) $t=1.67 \mathrm{~s}$; (c) $t=2.34 \mathrm{~s}$; (d) $t=3.02 \mathrm{~s}$ 

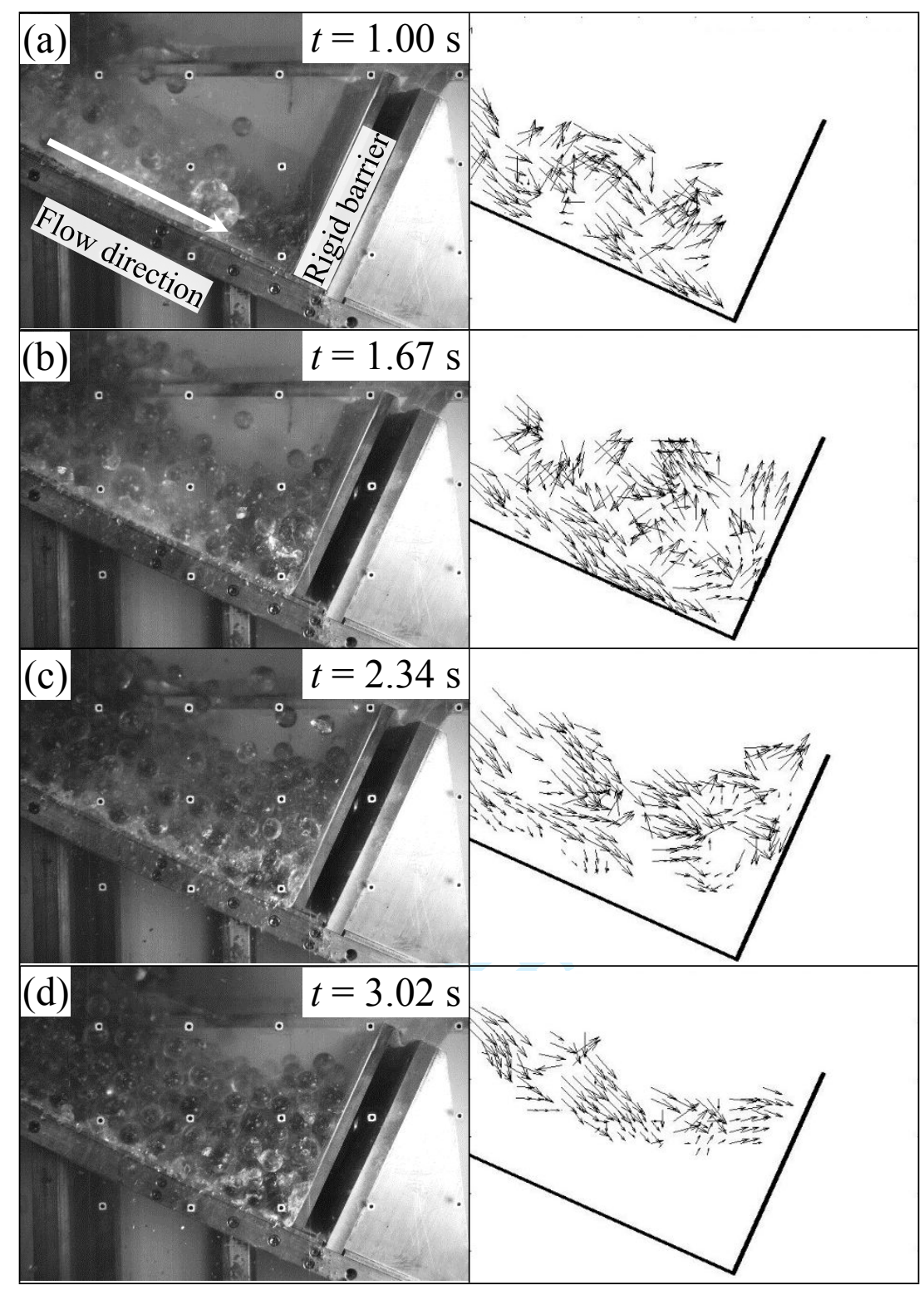

Fig. 4. Side view of the observed kinematics and velocity fields from particle image velocimetry for bi-disperse flow $\left(\delta_{\mathrm{s}} / \delta_{1}=0.56\right.$ ): (a) $t=1.00 \mathrm{~s}$; (b) $t=1.67 \mathrm{~s}$; (c) $t=2.34 \mathrm{~s}$; (d) $t=3.02 \mathrm{~s}$ 


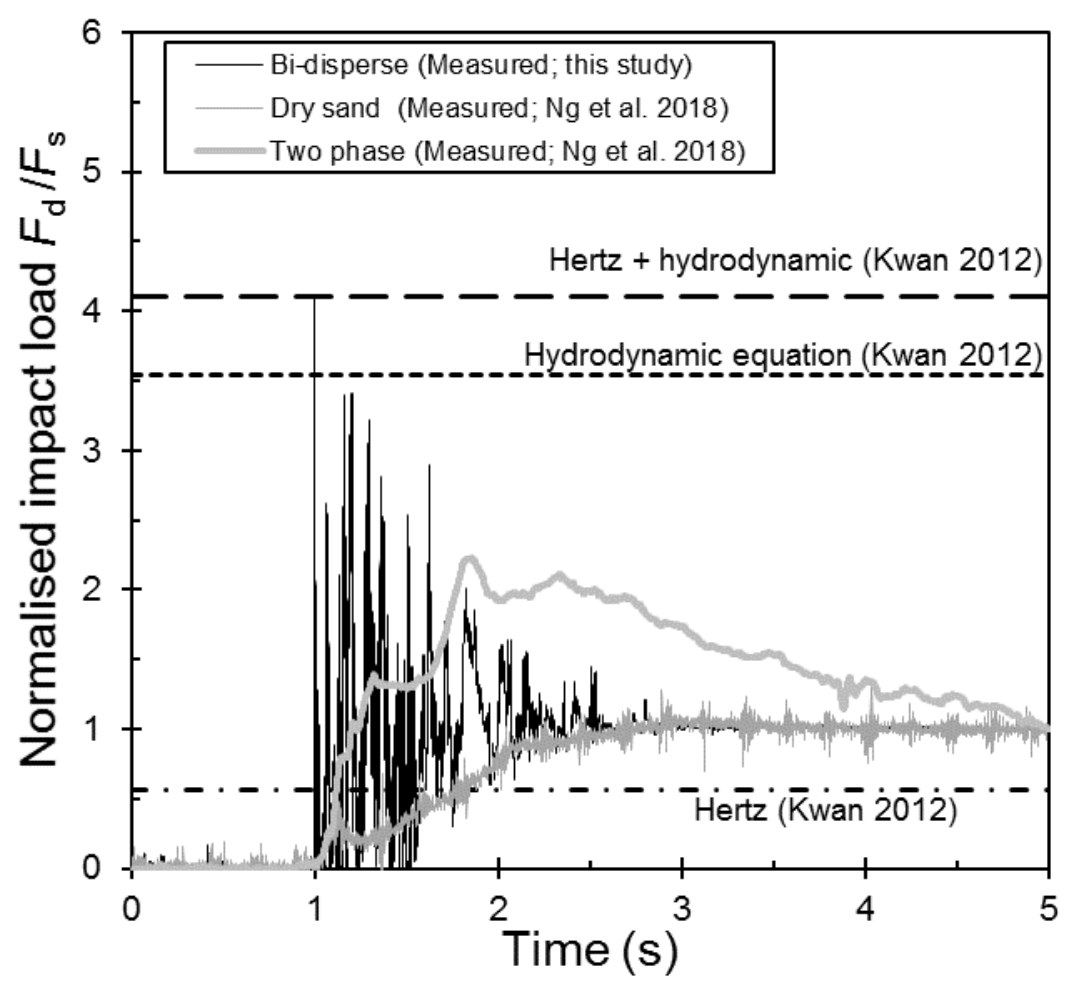

(a)

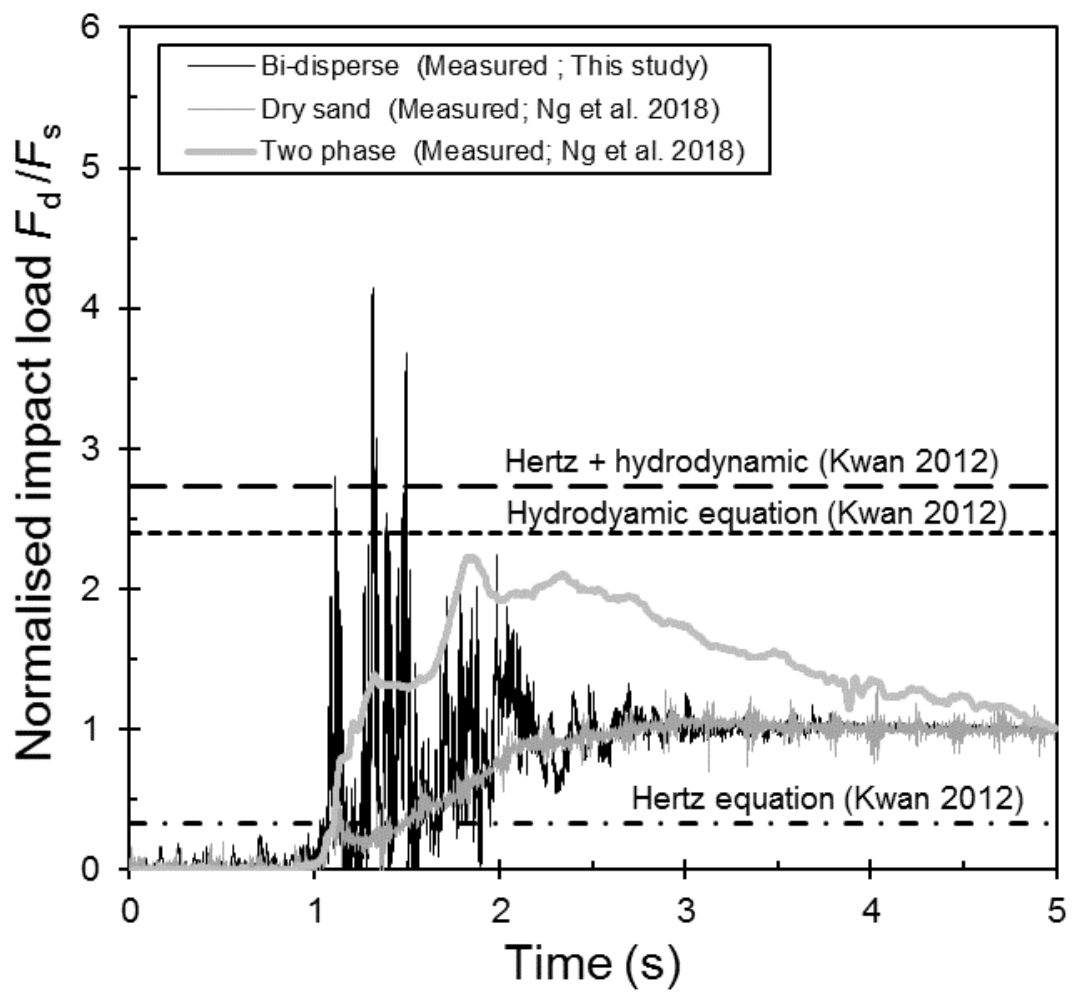

(b) 


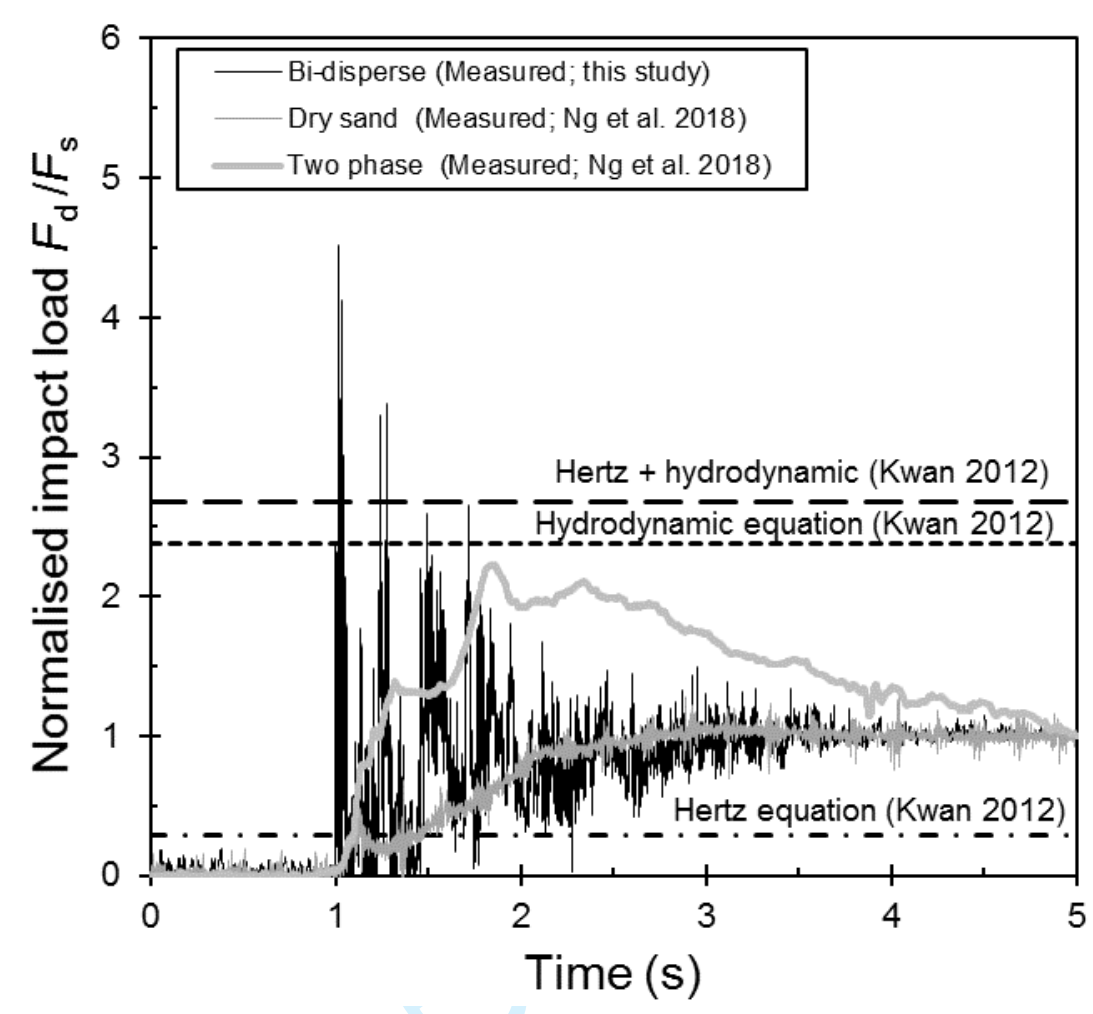

(c)

Fig. 5. Normalised impact load time-history on rigid barrier: (a) $\delta_{\mathrm{s}} / \delta_{1}=0.08$; (b) $\delta_{\mathrm{s}} / \delta_{1}=0.26$; (c) $\delta_{\mathrm{s}} / \delta_{1}=0.56$ 


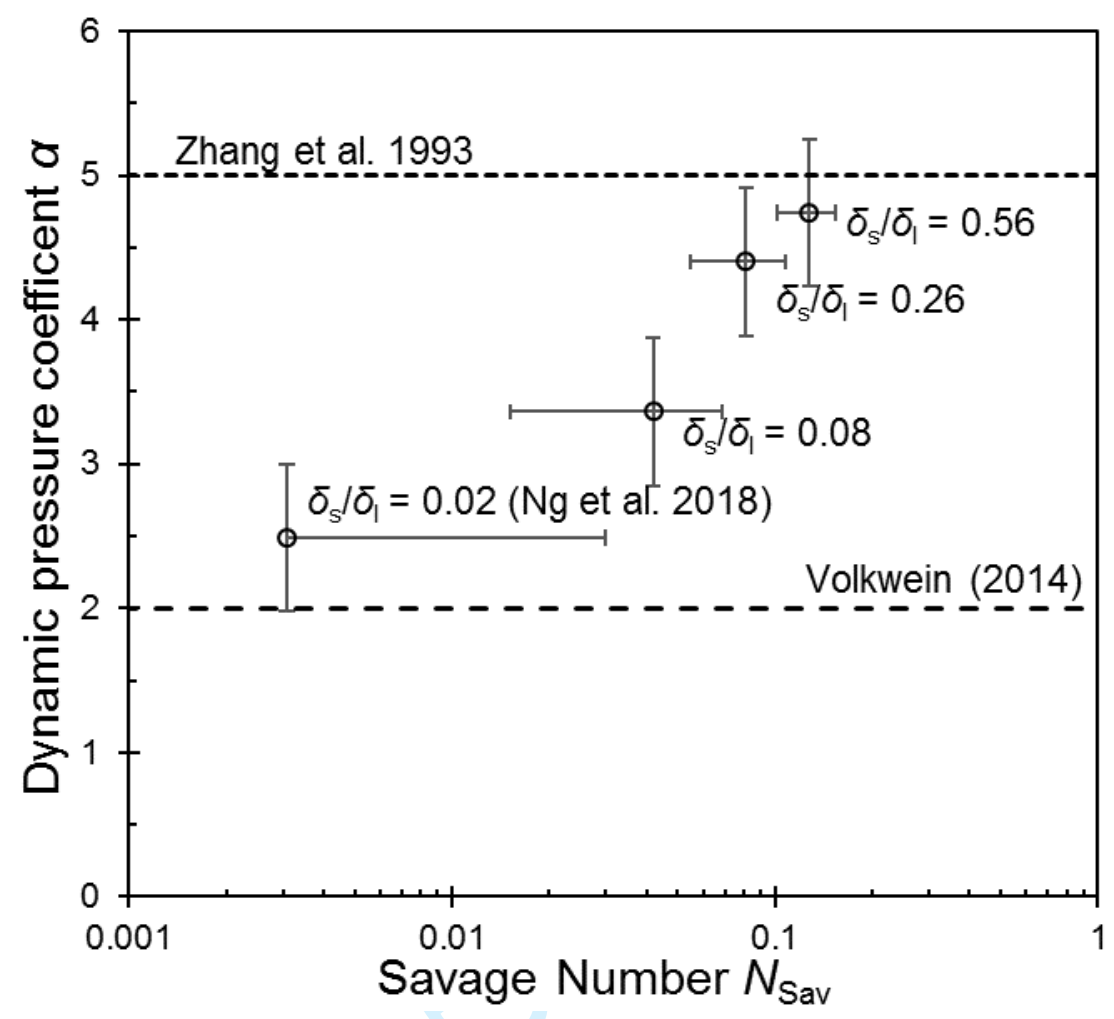

Fig. 6. Effects of grain-stresses on dynamic pressure coefficient for rigid barrier 


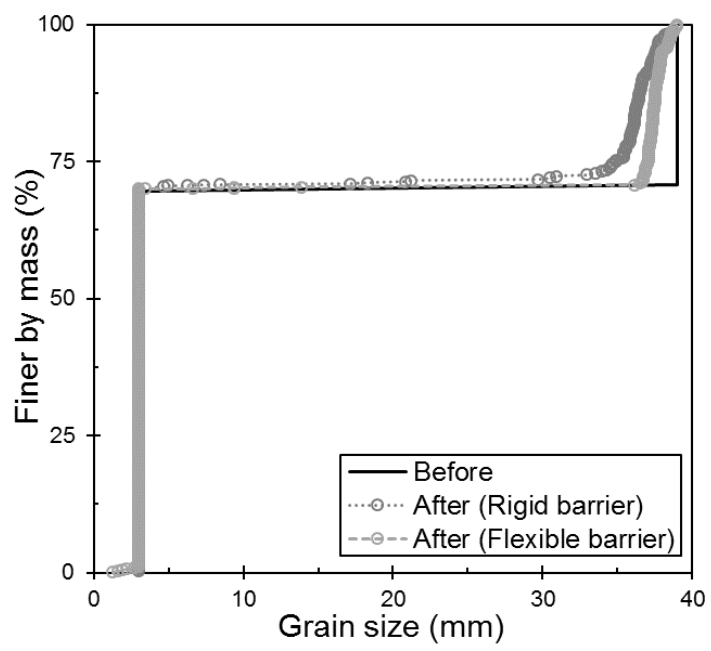

(a)

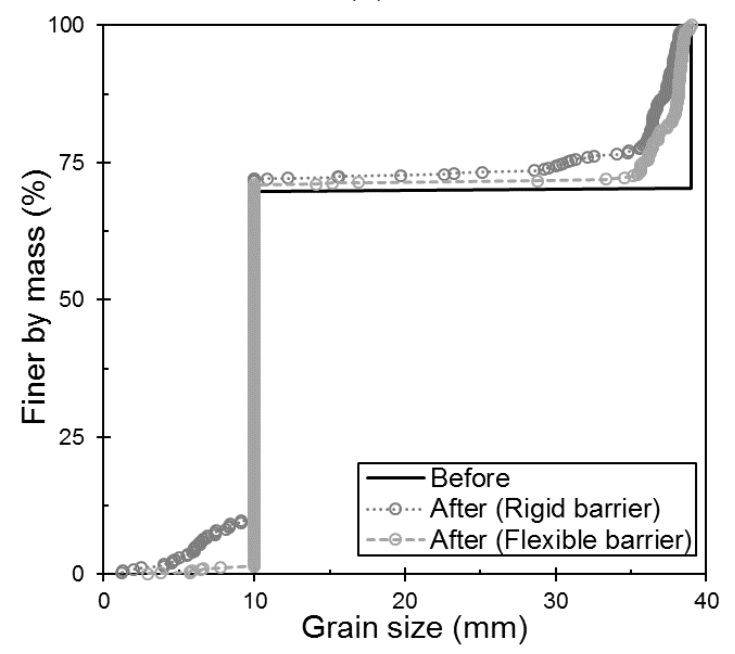

(b)

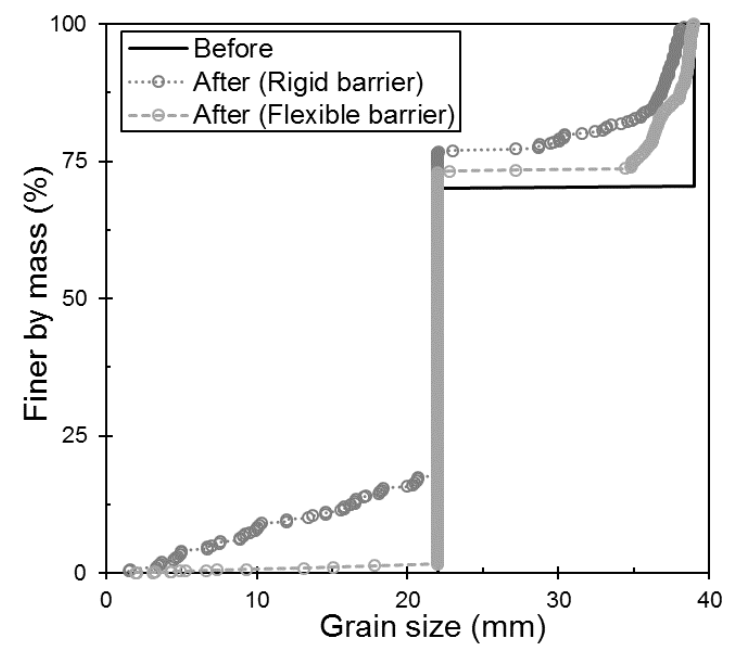

(c)

Fig. 7. Measured particle size distributions (PSD) for rigid and flexible barrier: (a) $\delta_{\mathrm{s}} / \delta_{1}=0.08$; (b) $\delta_{\mathrm{s}} / \delta_{1}=0.26 ;$ (c) $\delta_{\mathrm{s}} / \delta_{1}=0.56$ 


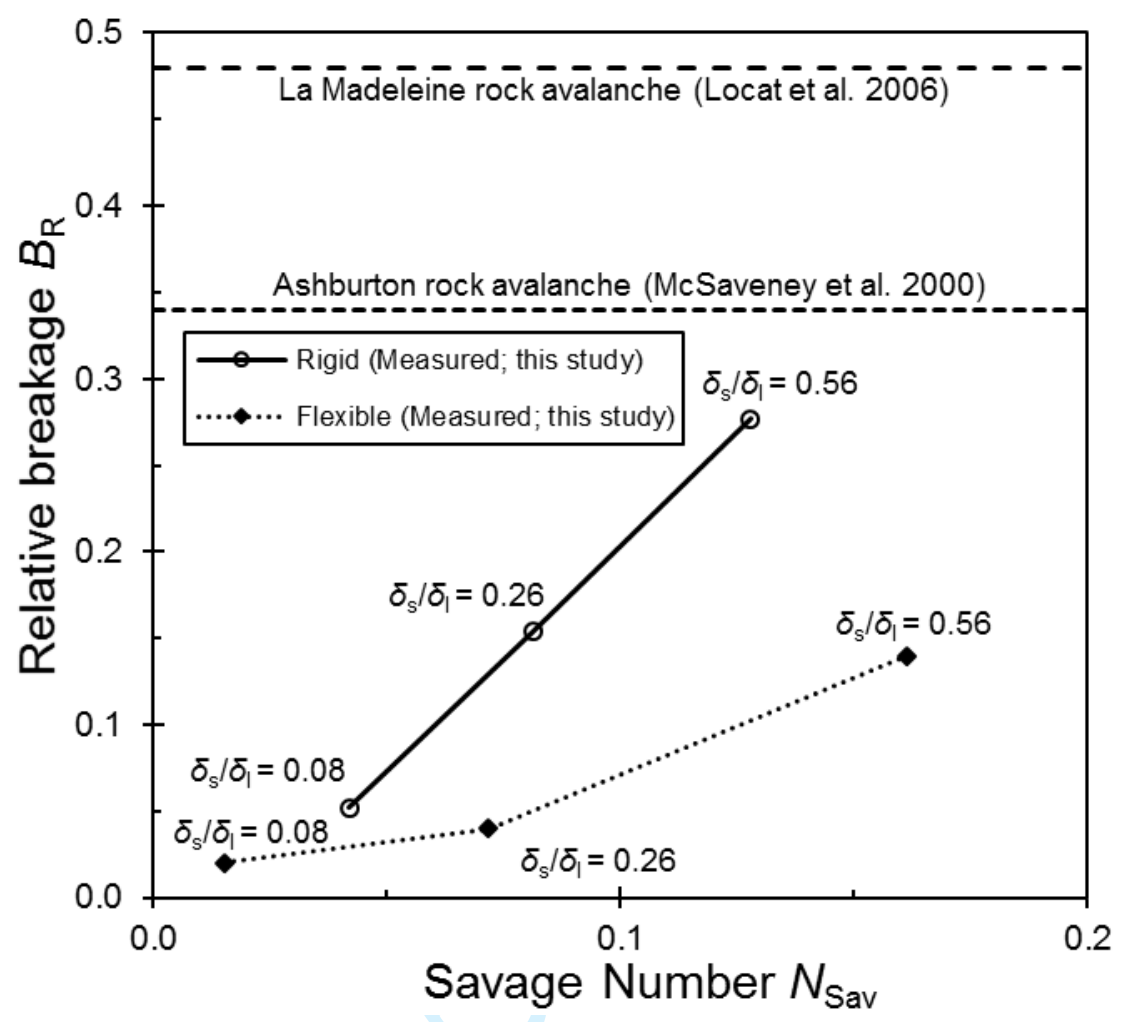

Fig. 8. Influence between Savage Number $N_{\text {Sav }}$ and relative breakage $B_{\mathrm{R}}$ 


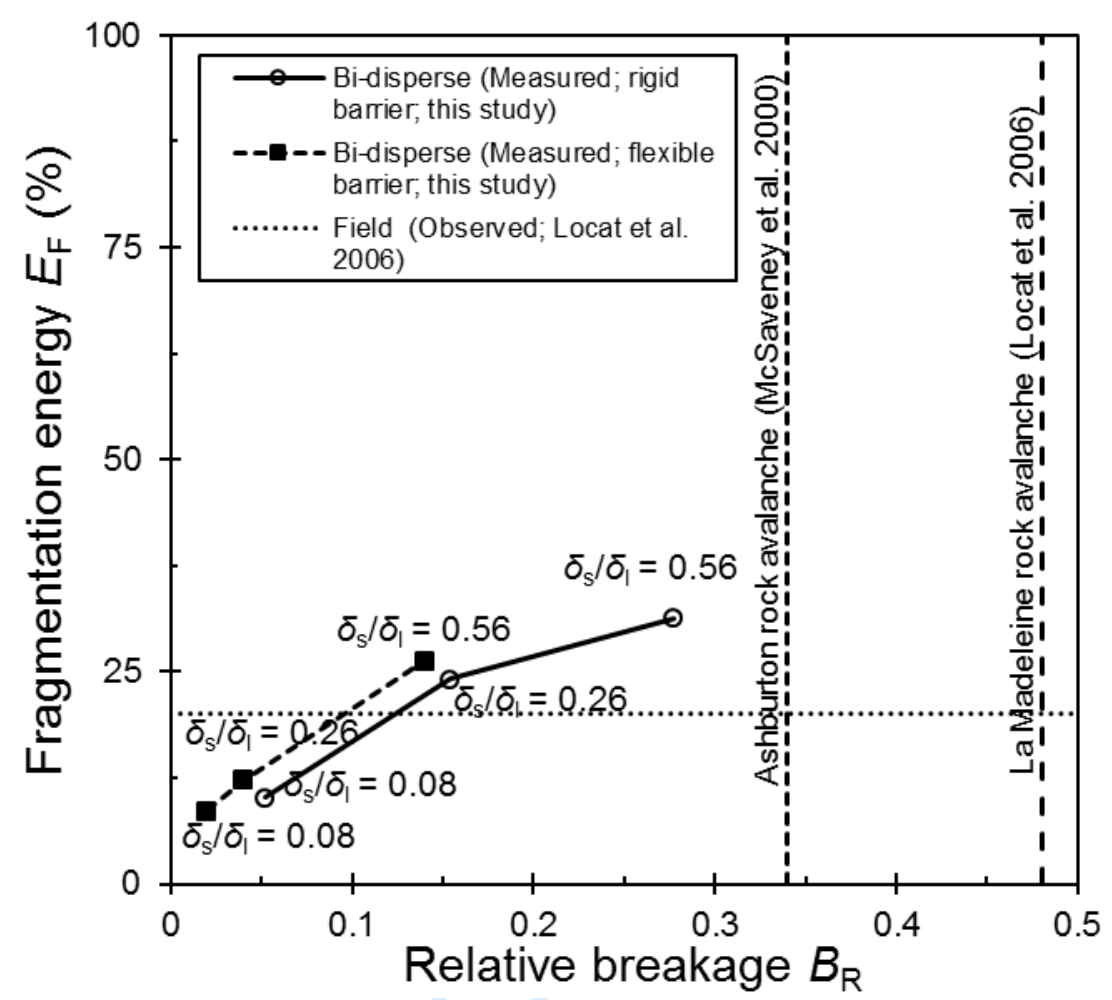

Fig. 9. Relationship between fragmentation energy $E_{\mathrm{F}}$ and relative breakage $B_{\mathrm{R}}$ 
Table 1. Summary of relevant centrifuge scaling laws

\begin{tabular}{|c|c|c|}
\hline Parameter & Dimension & Scaling law (Model / Prototype) \\
\hline Length & $\mathrm{L}$ & $1 / \mathrm{N}$ \\
\hline Mass & $\mathrm{M}$ & $1 / \mathrm{N}^{3}$ \\
\hline Inertia time & $\mathrm{T}$ & $1 / \mathrm{N}$ \\
\hline Shear strain rate & $\mathrm{T}^{-1}$ & $\mathrm{~N}$ \\
\hline Velocity & $\mathrm{LT}^{-1}$ & 1 \\
\hline Acceleration & $\mathrm{LT}^{-2}$ & $1 / \mathrm{N}$ \\
\hline Volume & $\mathrm{L}^{3}$ & $1 / \mathrm{N}^{3}$ \\
\hline Density & $\mathrm{ML}^{-3}$ & 1 \\
\hline Force & $\mathrm{MLT}^{-2}$ & $1 / \mathrm{N}^{2}$ \\
\hline Kinetic energy & $\mathrm{ML}^{2} \mathrm{~T}^{-2}$ & $1 / \mathrm{N}^{3}$ \\
\hline
\end{tabular}

Table 2. Test programme and flow characterisation

\begin{tabular}{|c|c|c|c|c|c|c|c|c|c|}
\hline \multirow{2}{*}{$\begin{array}{l}\text { Test } \\
\text { ID }\end{array}$} & \multirow{2}{*}{$\begin{array}{c}\text { Barrier } \\
\text { type }\end{array}$} & \multicolumn{2}{|c|}{$\begin{array}{c}\text { Model particle } \\
\text { diameter (prototype) } \\
\mathrm{mm}\end{array}$} & \multirow{2}{*}{$\begin{array}{c}\text { Normalised } \\
\text { diameter } \\
\delta_{\mathrm{s} /} \delta_{1}\end{array}$} & \multirow{2}{*}{$\begin{array}{c}\text { Flow } \\
\text { depth } h \\
\text { (prototype) } \\
\text { (m) }\end{array}$} & \multirow{2}{*}{$\begin{array}{c}\text { Initial } \\
\text { bulk } \\
\text { density } \\
\rho_{\text {bulk }} \\
\left(\mathrm{kg} / \mathrm{m}^{3}\right)\end{array}$} & \multirow{2}{*}{$\begin{array}{c}\text { Flow } \\
\text { velocity } \\
v \\
(\mathrm{~m} / \mathrm{s})\end{array}$} & \multirow{2}{*}{$\begin{array}{c}\text { Froude } \\
\text { Number } \\
\quad F r\end{array}$} & \multirow{2}{*}{$\begin{array}{c}\text { Savage } \\
\text { Number } \\
N_{\text {Sav }}\end{array}$} \\
\hline & & $\begin{array}{c}\text { Large } \\
\text { particle } \\
\delta_{1} \\
\end{array}$ & $\begin{array}{c}\text { Small } \\
\text { particle } \\
\delta_{\mathrm{s}} \\
\end{array}$ & & & & & & \\
\hline R3 & \multirow{3}{*}{ Rigid } & \multirow{6}{*}{$\begin{array}{c}39 \\
(874)\end{array}$} & $3(67)$ & 0.08 & $\begin{array}{c}0.05 \\
(1.21)\end{array}$ & 1390 & 12.7 & 3.87 & 0.04 \\
\hline $\mathrm{R} 10$ & & & $10(224)$ & 0.26 & $\begin{array}{c}0.08 \\
(1.79)\end{array}$ & 1348 & 9.6 & 2.40 & 0.08 \\
\hline $\mathrm{R} 22$ & & & $22(492)$ & 0.56 & $\begin{array}{c}0.11 \\
(2.42)\end{array}$ & 1240 & 8.6 & 1.84 & 0.13 \\
\hline F3 & \multirow{3}{*}{ Flexible } & & $3(67)$ & 0.08 & $\begin{array}{c}0.07 \\
(1.49)\end{array}$ & 1390 & 10.5 & 2.88 & 0.02 \\
\hline F10 & & & $10(224)$ & 0.26 & $\begin{array}{c}0.10 \\
(2.20) \\
\end{array}$ & 1348 & 12.2 & 2.76 & 0.07 \\
\hline $\mathrm{F} 22$ & & & $22(492)$ & 0.56 & $\begin{array}{c}0.10 \\
(2.28) \\
\end{array}$ & 1240 & 8.8 & 1.96 & 0.16 \\
\hline
\end{tabular}

Table 3. Mass measurement and relative breakage of particles before and after the test

\begin{tabular}{|c|c|c|c|c|c|}
\hline \multicolumn{2}{|c|}{ Test ID } & Mass before test $(\mathrm{kg})$ & Mass after test $(\mathrm{kg})$ & Percentage of lost mass (\%) & Relative breakage $B_{\mathrm{R}}$ \\
\hline \multirow{2}{*}{$\mathrm{R} 3$} & $\delta_{1}$ & 6.94 & 6.70 & 3.6 & \multirow{2}{*}{0.05} \\
\hline & $\delta_{\mathrm{s}}$ & 16.20 & 16.17 & 0.2 & \\
\hline \multirow{2}{*}{$\mathrm{R} 10$} & $\delta_{1}$ & 6.94 & 6.61 & 5.0 & \multirow{2}{*}{0.12} \\
\hline & $\delta_{\mathrm{s}}$ & 16.20 & 16.05 & 0.9 & \\
\hline \multirow{2}{*}{ R22 } & $\delta_{1}$ & 6.94 & 6.07 & 14.3 & \multirow{2}{*}{0.28} \\
\hline & $\delta_{\mathrm{s}}$ & 16.20 & 16.01 & 1.2 & \\
\hline \multirow{2}{*}{ F3 } & $\delta_{1}$ & 6.94 & 6.81 & 1.9 & \multirow{2}{*}{0.02} \\
\hline & $\delta_{\mathrm{s}}$ & 16.20 & 16.12 & 0.5 & \\
\hline \multirow{2}{*}{ F10 } & $\delta_{1}$ & 6.94 & 6.73 & 3.1 & \multirow{2}{*}{0.04} \\
\hline & $\delta_{\mathrm{s}}$ & 16.20 & 16.12 & 0.5 & \\
\hline \multirow{2}{*}{$\mathrm{F} 22$} & $\delta_{1}$ & 6.94 & 6.32 & 9.8 & \multirow{2}{*}{0.14} \\
\hline & $\delta_{\mathrm{s}}$ & 16.20 & 16.08 & 0.7 & \\
\hline
\end{tabular}

